\title{
Behavior of the collective rotor in wobbling motion
}

\author{
E. Streck, ${ }^{1}$ Q. B. Chen, ${ }^{1, *}$ N. Kaiser, ${ }^{1, \dagger}$ and Ulf-G. Meißner ${ }^{2,3,4, \ddagger}$ \\ ${ }^{1}$ Physik-Department, Technische Universität München, D-85747 Garching, Germany \\ ${ }^{2}$ Helmholtz-Institut für Strahlen- und Kernphysik and Bethe Center for Theoretical Physics, Universität Bonn, D-53115 Bonn, Germany \\ ${ }^{3}$ Institute for Advanced Simulation, Institut für Kernphysik, Jülich Center for Hadron Physics and JARA-HPC, \\ Forschungszentrum Jülich, D-52425 Jülich, Germany \\ ${ }^{4}$ Ivane Javakhishvili Tbilisi State University, 0186 Tbilisi, Georgia
}

(Received 9 May 2018; revised manuscript received 4 September 2018; published 12 October 2018)

\begin{abstract}
The behavior of the collective rotor in wobbling motion is investigated within the particle-rotor model for the nucleus ${ }^{135} \mathrm{Pr}$ by transforming the wave functions from the $K$ representation to the $R$ representation. After reproducing the experimental energy spectra and wobbling frequencies, the evolution of the wobbling mode in ${ }^{135} \mathrm{Pr}$, from transverse at low spins to longitudinal at high spins, is illustrated by the distributions of the total angular momentum in the intrinsic reference frame (azimuthal plot). Finally, the coupling schemes of the angular momenta of the rotor and the high- $j$ particle for transverse and longitudinal wobbling are obtained from the analysis of the probability distributions of the rotor angular momentum ( $R$ plots $)$ and their projections onto the three principal axes ( $K_{R}$ plots).
\end{abstract}

DOI: 10.1103/PhysRevC.98.044314

\section{INTRODUCTION}

As a quantum-mechanical complex many-body system, an atomic nucleus can possess a wide variety of shapes in its ground and excited states. The shapes may range from spherical to deformed (quadrupole, octupole, etc.) and even more exotic shapes, such as superdeformed and tetrahedral are possible. At the same time, the atomic nucleus can exhibit various modes of collective excitations. Obviously, the modes of collective motion are strongly correlated with the nuclear shapes. For example, only a nucleus with triaxial deformation can possibly have chiral rotation [1] or wobbling motion [2].

The wobbling motion was first proposed by Bohr and Mottelson in the 1970s [2]. It occurs in the case when the rotation of a triaxial nucleus about the principal axis with the largest moment of inertia (MoI) is quantum mechanically disturbed by rotations about the other two principal axes, and hence it precesses and wobbles around the axis with the largest MoI. The energy spectra related to the wobbling motion are called wobbling bands, and these consist of sequences of $\Delta I=2$ rotational bands built on different wobbling-phonon excitations [2].

The excitation spectrum of the wobbling motion is characterized by the wobbling frequency. For the originally predicted wobbler (a triaxial rotor built up by an even-even nucleus) [2], the wobbling frequency increases with spin. For an odd-mass nucleus, the triaxial rotor is coupled with a high$j$ quasiparticle, and in this case two different wobbling modes were proposed by Frauendorf and Dönau [3]. One of them

\footnotetext{
*qbchen@pku.edu.cn

†nkaiser@ph.tum.de

${ }^{\ddagger}$ meissner@hiskp.uni-bonn.de
}

is called longitudinal wobbling, in which the quasiparticle angular momentum is parallel to the principal axis with the largest MoI. The other one is named transverse wobbling, since the quasiparticle angular momentum is perpendicular to the principal axis with the largest MoI. According to Ref. [3] using the frozen alignment approximation, the wobbling frequency

$$
\hbar \Omega_{w}=\frac{j}{\mathcal{J}_{1}}\left[\left(1+\frac{I}{j}\left(\frac{\mathcal{J}_{1}}{\mathcal{J}_{3}}-1\right)\right)\left(1+\frac{I}{j}\left(\frac{\mathcal{J}_{1}}{\mathcal{J}_{2}}-1\right)\right)\right]^{1 / 2}
$$

of a longitudinal wobbler increases, while that of a transverse wobbler decreases with increasing spin $I$. In Eq. (1), $\mathcal{J}_{k}$ are the three principal MoIs, and $j$ is the angular momentum of the aligned particle. The short axis in the transverse wobbling and the intermediate axis in the longitudinal wobbling are named as the one-axis. Note that transverse wobbling occurs in a situation when the particle angular momentum is aligned along the (short) one-axis, which is perpendicular to the axis with the largest $\mathrm{MoI} \mathcal{J}_{2}$, with the two-axis being of intermediate length. Transverse wobbling can be realized since the additional particle can invert the ordering of the effective MoIs of the one- and two-axes. This property is obtained from microscopic calculations using random phase approximation (RPA) in Refs. [4,5], an analysis in the particlerotor model [3,5], and the collective Hamiltonian method [6].

Wobbling bands have been reported in the mass region $A \approx 160$ for the isotopes ${ }^{161} \mathrm{Lu}[7],{ }^{163} \mathrm{Lu}[8,9],{ }^{165} \mathrm{Lu}[10]$, ${ }^{167} \mathrm{Lu}[11]$, and ${ }^{167} \mathrm{Ta}[12]$, in the mass region $A \approx 110$ for ${ }^{112} \mathrm{Ru}[13]$ and ${ }^{114} \mathrm{Pd}[14]$, and recently in the mass region $A \approx 130$ for ${ }^{135} \operatorname{Pr}[15]$ and ${ }^{133} \mathrm{La} \mathrm{[16]}$.

Interestingly, the isotope ${ }^{135} \mathrm{Pr}$ does not only possess the transverse wobbling mode, but also exhibits a transition from 
transverse to longitudinal wobbling [15]. Hence, ${ }^{135} \mathrm{Pr}$ is an excellent candidate for understanding the wobbling motion and has attracted a lot of theoretical attention. These studies can be briefly summarized as follows:

(i) In Refs. $[3,15]$, the tilted axis cranking (TAC) model with Strutinsky shell corrections and the particle-rotor model (PRM) were employed to confirm the wobbling nature of the experimental energy spectra and the electromagnetic transition probabilities.

(ii) In Ref. [17], the multi-quasiparticle triaxial projected shell model (TPSM) approach was used to extract the probabilities of various projected configurations in the wave functions of the yrast and the wobbling states.

(iii) In Ref. [6], a collective Hamiltonian method based on the TAC approach was applied to reveal the microscopic mechanisms underlying the variation of the wobbling frequency with spin and the transition from transverse to longitudinal wobbling.

(iv) In Ref. [18], the Holstein-Primakoff boson expansion was applied to the PRM to examine the stability of the wobbling motion.

(v) In Ref. [19], a time-dependent variational method, with coherent angular momentum states as variational states, was adopted to treat the PRM (specialized to a high- $j$ quasiparticle aligned rigidly with one principal axis) and to obtain analytical solutions for the energy spectra and electromagnetic transition probabilities.

However, still no attempt has been made to investigate the detailed structure of wave functions of the collective rotor in wobbling states. Taking ${ }^{135} \mathrm{Pr}$ as an example, we investigate in this paper the behavior of the collective rotor angular momentum in wobbling motion using the PRM.

For this purpose, one has to express the PRM wave function in terms of the weak-coupling basis [2,20], in which both $R$ (rotor angular momentum quantum number) and $K_{R}$ (projection on a principal axis) are good quantum numbers. This transformation gives the $R$ representation. From the corresponding probability distributions one can derive (by summation) the $R$ plot and the $K_{R}$ plots.

Usually, the PRM wave functions are formulated in terms of the strong coupling basis [2,20], where the projection of the total spin onto the three-axis of the intrinsic frame is a good quantum number, denoted by $K$. In this $K$ representation, $R$ and $K_{R}$ do not appear explicitly. Therefore, in order to obtain the $R$ plot and the $K_{R}$ plots, one has to transform the PRM wave function from the $K$ representation to the $R$ representation. This technique has been applied for a long time to take into account $R$-dependent MoIs [2,21-26] or shape fluctuations of the rotor $[27,28]$ in the description of rotational spectra, or to calculate decay widths of proton emitters [29-32]. The probability distributions of the rotor angular momentum were also obtained before in an analysis of the rotational spectra of axially symmetric nuclei [23]. Here, it is employed for the first time to investigate the detailed wave function structure of the collective rotor in the wobbling motion of a triaxial nucleus.

\section{THEORETICAL FRAMEWORK}

\section{A. Particle-rotor Hamiltonian}

The total Hamiltonian of the PRM takes the form $[2,20]$

$$
\hat{H}_{\text {PRM }}=\hat{H}_{\text {coll }}+\hat{H}_{\text {intr }}
$$

with $\hat{H}_{\text {coll }}$ the collective rotor Hamiltonian

$$
\begin{aligned}
\hat{H}_{\mathrm{coll}} & =\sum_{k=1}^{3} \frac{\hat{R}_{k}^{2}}{2 \mathcal{J}_{k}} \\
& =\sum_{k=1}^{3} \frac{\left(\hat{I}_{k}-\hat{j}_{k}\right)^{2}}{2 \mathcal{J}_{k}},
\end{aligned}
$$

where the index $k=1,2,3$ denotes the three principal axes of the body-fixed frame. Here, $\hat{R}_{k}$ and $\hat{I}_{k}$ are the angular momentum operators of the collective rotor and the total nucleus, and $\hat{j}_{k}$ is the angular momentum operator of a valence nucleon. Moreover, the parameters $\mathcal{J}_{k}$ are the three principal MoIs. When calculating matrix elements of $\hat{H}_{\text {coll }}$, the $R$ representation is most conveniently used for its form in Eq. (3), while Eq. (4) is preferable in the $K$ representation.

The intrinsic Hamiltonian $\hat{H}_{\text {intr }}$ describes a single valence nucleon in a high- $j$ shell

$$
\hat{H}_{\text {intr }}= \pm \frac{1}{2} C\left\{\cos \gamma\left(\hat{j}_{3}^{2}-\frac{j(j+1)}{3}\right)+\frac{\sin \gamma}{2 \sqrt{3}}\left(\hat{j}_{+}^{2}+\hat{j}_{-}^{2}\right)\right\}
$$

where \pm refers to a particle or a hole state. The angle $\gamma$ serves as the triaxial deformation parameter and the coefficient $C$ is proportional to the quadrupole deformation parameter $\beta$. We take in the present work the same form of $C$ as in Ref. [33].

\section{B. Basis transformation from $K$ representation to $R$ representation}

As mentioned in the Introduction, the PRM Hamiltonian (2) is usually solved by diagonalization in the strong-coupling basis ( $K$ representation) $[2,20]$

$$
\begin{aligned}
|I M K j \Omega\rangle= & \sqrt{\frac{2 I+1}{16 \pi^{2}}}\left[D_{M K}^{I}(\omega)|j \Omega\rangle\right. \\
& \left.+(-1)^{I-j} D_{M-K}(\omega)|j-\Omega\rangle\right],
\end{aligned}
$$

where $I$ denotes the total angular momentum quantum number of the odd-mass nuclear system (rotor plus particle) and $M$ is the projection onto the three-axis of the laboratory frame. Furthermore, $\Omega$ is the three-axis component of the particle angular momentum $j$ in the intrinsic frame, and $D_{M K}^{I}(\boldsymbol{\omega})$ are the usual Wigner functions, depending on three Euler angles $\boldsymbol{\omega}=\left(\psi^{\prime}, \theta^{\prime}, \phi^{\prime}\right)$. Under the requirement of the $\mathrm{D}_{2}$ symmetry of a triaxial nucleus [2], $K$ and $\Omega$ take the values: $K=-I, \ldots, I, \Omega=-j, \ldots, j, K-\Omega \geqslant 0$ and even; and if $K-\Omega=0, K=\Omega>0$.

As seen in the $K$ representation (6), the rotor angular momentum $R$ does not appear explicitly. In order to obtain the wave function of the rotor in the $R$ representation, one has to transform the basis. The details of this transformation can be found in Refs. [26,30]. Here, we outline the main ingredients. 
The wave function of the total nuclear system in the laboratory frame can be expressed in the $R$ representation as

$$
|I M j R \tau\rangle=\sum_{m, M_{R}}\left\langle j m R M_{R} \mid I M\right\rangle|j m\rangle \otimes\left|R M_{R} \tau\right\rangle,
$$

where $m$ and $M_{R}$ are the projections of $\boldsymbol{j}$ and $\boldsymbol{R}$ on the three-axis of the laboratory frame. Obviously, the appearance of Clebsch-Gordan coefficients requires $M=m+M_{R}$, and the values of $R$ must satisfy the triangular condition $|I-j| \leqslant$ $R \leqslant I+j$ of angular momentum coupling. At the moment, the additional quantum number $\tau$, related to the projection of $R$ on a body-fixed axis, is not yet specified. Now we perform the transformation from the $R$ representation to the $K$ representation.

In the $K$ representation, the quantum number $\tau$ is identified with the projection $K_{R}$ of $R$ on a principal axis. Making use of Wigner functions, the wave functions of the particle and the rotor in Eq. (7) can be written as

$$
\begin{aligned}
|j m\rangle= & \sum_{\Omega=-j}^{j} D_{m \Omega}^{j}(\omega)|j \Omega\rangle, \\
\left|R M_{R} K_{R}\right\rangle= & \sqrt{\frac{2 R+1}{16 \pi^{2}\left(1+\delta_{K_{R} 0}\right)}} \\
& \times\left[D_{M_{R} K_{R}}^{R}(\omega)+(-1)^{R} D_{M_{R}-K_{R}}^{R}(\omega)\right],
\end{aligned}
$$

where $K_{R}$ is an even integer ranging from 0 to $R$, with $K_{R}=0$ is excluded for odd $R$. Both restrictions come from the $D_{2}$ symmetry of a triaxial nucleus [2]. Note that for an axially symmetric nucleus, $R$ can only take even integer values since $K_{R}$ must be zero.

Substituting Eqs. (8) and (9) into Eq. (7), one obtains

$$
\left|I M j R K_{R}\right\rangle=\sum_{K, \Omega} A_{j \Omega, R K_{R}}^{I K}|I M K j \Omega\rangle
$$

with the expansion coefficients,

$$
A_{j \Omega, R K_{R}}^{I K}=\sqrt{\frac{2 R+1}{2 I+1}}\left\langle j \Omega R K_{R} \mid I K\right\rangle \sqrt{1+\delta_{K_{R} 0}},
$$

determined by Clebsch-Gordan coefficients (hence $K=$ $\left.K_{R}+\Omega\right)$.

Obviously, the transformation between the $K$ representation and the $R$ representation is an orthogonal transformation, and therefore the expansion coefficients satisfy

$$
\begin{aligned}
& \sum_{K, \Omega} A_{j \Omega, R K_{R}}^{I K} A_{j \Omega, R^{\prime} K_{R}^{\prime}}^{I K}=\delta_{R R^{\prime}} \delta_{K_{R} K_{R}^{\prime}}, \\
& \sum_{R, K_{R}} A_{j \Omega, R K_{R}}^{I K} A_{j \Omega^{\prime}, R K_{R}}^{I K^{\prime}}=\delta_{\Omega \Omega^{\prime}} \delta_{K K^{\prime}} .
\end{aligned}
$$

Due to this orthogonality property, the inverse transformation follows immediately as

$$
|I M K j \Omega\rangle=\sum_{R, K_{R}} A_{j \Omega, R K_{R}}^{I K}\left|I M j R K_{R}\right\rangle .
$$

To this end, we have successfully transformed the PRM basis functions from the $K$ representation to $R$ representation.
Equation (14) allows us also to calculate the matrix elements of the collective rotor Hamiltonian in the $K$ representation as

$$
\begin{aligned}
& \left\langle I M K^{\prime} j \Omega^{\prime}\left|\hat{H}_{\mathrm{coll}}\right| I M K j \Omega\right\rangle \\
& \quad=\sum_{R, K_{R}, K_{R}^{\prime}} A_{j \Omega^{\prime}, R K_{R}^{\prime}}^{I K^{\prime}}\left\langle I M j R K_{R}^{\prime}\left|\hat{H}_{\mathrm{coll}}\right| I M j R K_{R}\right\rangle A_{j \Omega, R K_{R}}^{I K} \\
& \quad=\sum_{R, K_{R}, K_{R}^{\prime}} A_{j \Omega^{\prime}, R K_{R}^{\prime}}^{I K^{\prime}}\left(\sum_{i} c_{K_{R}^{\prime}}^{R i} E_{R i} c_{K_{R}}^{R i}\right) A_{j \Omega, R K_{R}}^{I K}
\end{aligned}
$$

where the energies $E_{R i}$ and corresponding expansion coefficients $c_{K_{R}}^{R i}$ ( $i$ labels the different eigenstates) are obtained by diagonalizing the collective rotor Hamiltonian $\hat{H}_{\text {coll }}$ in the basis $\left|R M_{R} K_{R}\right\rangle$ introduced in Eq. (9):

$$
\begin{aligned}
\hat{H}_{\mathrm{coll}}\left|R M_{R} i\right\rangle & =E_{R i}\left|R M_{R} i\right\rangle, \\
\left|R M_{R} i\right\rangle & =\sum_{K_{R}} c_{K_{R}}^{R i}\left|R M_{R} K_{R}\right\rangle .
\end{aligned}
$$

In such a calculation, $R$-dependent MoIs can be easily implemented in the PRM to obtain a better description of high spin states [2,21-24,26]. The main focus of the present work is on the probability distributions of the rotor angular momentum derived from the transformation (14).

\section{C. $R$ plot and $K_{R}$ plot}

With the above preparations, the PRM eigenfunctions can be expressed as

$$
\begin{aligned}
|I M\rangle= & \sum_{K, \Omega} d_{K, \Omega}|I M K j \Omega\rangle \\
= & \sum_{K, \Omega} d_{K, \Omega} \sum_{R, K_{R}} A_{j \Omega, R K_{R}}^{I K} \\
& \times \sum_{m, M_{R}}\left\langle j m R M_{R} \mid I M\right\rangle\left|R M_{R} K_{R}\right\rangle|j m\rangle,
\end{aligned}
$$

where the (real) expansion coefficients $d_{K, \Omega}$ are obtained by solving the total PRM Hamiltonian $\hat{H}_{\text {coll }}+\hat{H}_{\text {intr }}$ in Eq. (2). Hence, the probabilities for given $R$ and $K_{R}$ are calculated as

$$
P_{R, K_{R}}=\left(\sum_{K, \Omega} d_{K, \Omega} A_{j \Omega, R K_{R}}^{I K}\right)^{2},
$$

and they satisfy the normalization condition

$$
\sum_{R, K_{R}} P_{R, K_{R}}=1
$$

The $R$ plot consists of the summed probabilities

$$
P_{R}=\sum_{K_{R}} P_{R, K_{R}}
$$

whereas in the $K_{R}$ plot the probabilities are summed differently

$$
P_{K_{R}}=\sum_{R} P_{R, K_{R}} .
$$


Moreover, the expectation value of the squared angular momentum operator $\hat{R}_{3}^{2}$ follows as

$$
\left\langle I M\left|\hat{R}_{3}^{2}\right| I M\right\rangle=\sum_{R, K_{R}} K_{R}^{2} P_{R, K_{R}} .
$$

\section{Azimuthal plot}

In this work, we want to illustrate the angular momentum geometry of the wobbling motion by a profile on the $(\theta, \varphi)$ unit sphere, called azimuthal plot [34,35]. Here, $(\theta, \varphi)$ are the orientation angles of the angular momentum vector $\boldsymbol{I}$ (expectation value with $M=I$ ) with respect to the intrinsic frame. The polar angle $\theta$ is the angle between $\boldsymbol{I}$ and the threeaxis, whereas the azimuthal angle $\varphi$ is the angle between the projection of $\boldsymbol{I}$ on the 12-plane and the one-axis. The profiles can be obtained by relating the orientation angles $(\theta, \varphi)$ to the Euler angles $\boldsymbol{\omega}=\left(\psi^{\prime}, \theta, \pi-\varphi\right)[1,34]$, where the $z$ axis in the laboratory frame is chosen along $\boldsymbol{I}$. The profiles are calculated from the PRM eigenfunctions (18) as

$$
\begin{aligned}
\mathcal{P}(\theta, \varphi) & \\
= & 2 \pi \sum_{\Omega_{p}} \mid \sum_{K, \Omega} d_{K, \Omega} \sqrt{\frac{2 I+1}{16 \pi^{2}}}\left[D_{I K}^{I}\left(\psi^{\prime}, \theta, \pi-\varphi\right) \delta_{\Omega_{p}, \Omega}\right. \\
& \left.+(-1)^{I-j} D_{I-K}^{I}\left(\psi^{\prime}, \theta, \pi-\varphi\right) \delta_{\Omega_{p},-\Omega}\right]\left.\right|^{2},
\end{aligned}
$$

where the factor $2 \pi$ comes from the integral over $\psi^{\prime}$. Note that $D_{I K}^{I}\left(\psi^{\prime}, \theta^{\prime}, \varphi^{\prime}\right) \sim e^{-i I \psi^{\prime}}$, and therefore the right-hand side of Eq. (25) is $\psi^{\prime}$ independent. The profiles $\mathcal{P}(\theta, \varphi)$ fulfill the normalization condition

$$
\int_{0}^{\pi} d \theta \sin \theta \int_{-\pi}^{\pi} d \varphi \mathcal{P}(\theta, \varphi)=1
$$

Due to the combination of Wigner functions required by the $D_{2}$ symmetry in Eq. (25), $\mathcal{P}(\theta, \varphi)$ fulfills the following relations: $\mathcal{P}(\theta, \varphi)=\mathcal{P}(\theta,-\varphi)=\mathcal{P}(\theta, \pi-\varphi)=\mathcal{P}(\pi-$ $\theta, \varphi)$. Therefore, the complete information is contained in the angle ranges $0 \leqslant \theta \leqslant \pi / 2$ and $0 \leqslant \varphi \leqslant \pi / 2$.

\section{NUMERICAL DETAILS}

In our calculation of the wobbling bands in ${ }^{135} \mathrm{Pr}$, the configuration of the proton is taken as $\pi\left(1 h_{11 / 2}\right)^{1}$. Following Refs. [3,6,15], the quadrupole deformation parameters of this configuration have the values $\beta=0.17$ and $\gamma=-26.0^{\circ}$. With this assignment of $\gamma$, the one-, two-, and three-axes are the short $(s)$, intermediate $(i)$, and long $(l)$ axes of the ellipsoid, respectively. The principal MoIs are taken as $\mathcal{J}_{1}$, $\mathcal{J}_{2}, \mathcal{J}_{3}=13.0,21.0,4.0 \hbar^{2} / \mathrm{MeV}[3,6]$. In this case, the $i$ axis is the axis with the largest MoI.

\section{RESULTS AND DISCUSSION}

\section{A. Energy spectra of wobbling bands}

In Fig. 1(a), the energy spectra of the yrast and wobbling bands calculated in the PRM are compared with the experimental data [15]. A similar figure has been given in Ref. [6],

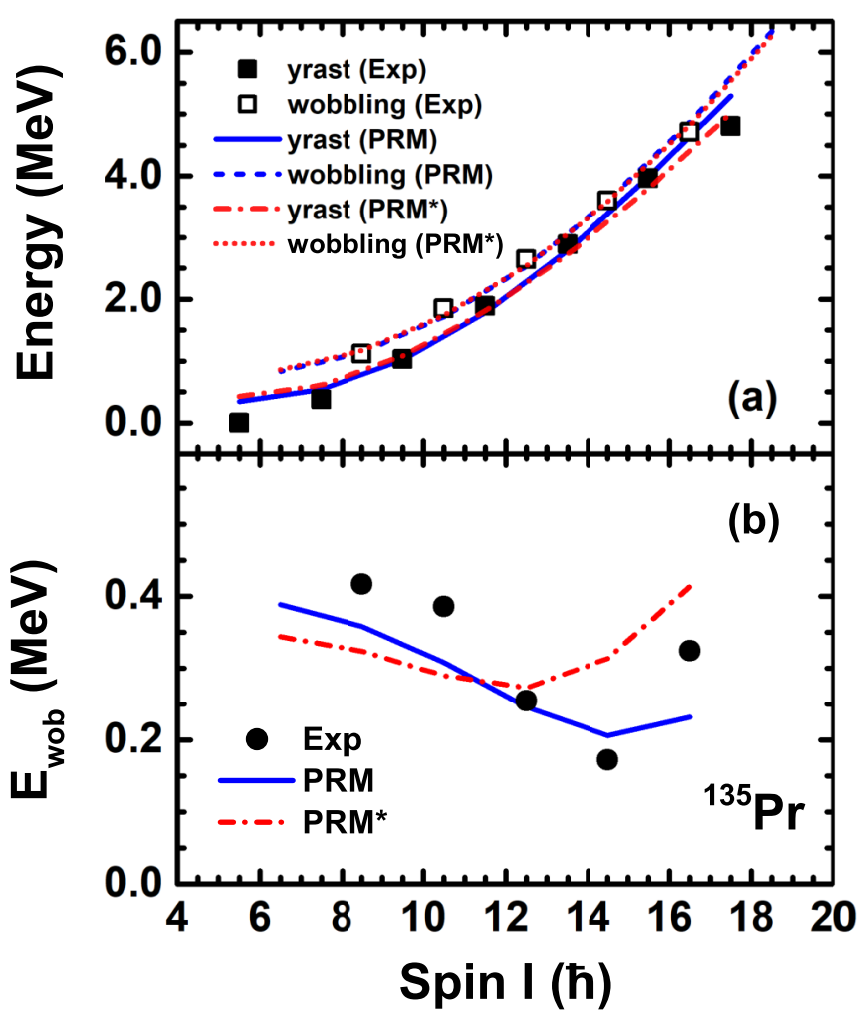

FIG. 1. Energy spectra of the yrast (zero phonon) and wobbling bands (one phonon) (a) and the corresponding wobbling frequency (b) in ${ }^{135} \mathrm{Pr}$ as functions of the total spin $I$ calculated without pairing (labeled as PRM) and with pairing (labeled as PRM*) in comparison to the experimental data of Ref. [15].

where the collective Hamiltonian method has been used. For both approaches, good agreement between the theoretical calculations and the data can be obtained.

From the energy spectra, the wobbling frequencies $E_{\text {wob }}(I)$ of the theoretical calculation and the data are extracted (as differences) and shown in Fig. 1(b) as a function of spin $I$. In the region $I \leqslant 14.5 \hbar$, both the theoretical and experimental wobbling frequencies decrease with spin, which provides evidence for transverse wobbling motion. At higher spin $(I \geqslant 14.5 \hbar)$, the experimental wobbling frequency shows an increasing trend, which indicates that the wobbling mode changes from transverse to longitudinal [15]. The PRM calculations can reproduce this transition well. Here, one should note that the ratio of $\mathcal{J}_{1}$ and $\mathcal{J}_{2}$ is essential for the existence of the transverse wobbling mode [3,5]. We fix $\mathcal{J}_{2}=21 \hbar^{2} / \mathrm{MeV}$ and $\mathcal{J}_{3}=4 \hbar^{2} / \mathrm{MeV}$ and change $\mathcal{J}_{1}$ in steps of $1 \hbar^{2} / \mathrm{MeV}$ around $13 \hbar^{2} / \mathrm{MeV}$, until the transverse wobbling band ends at $16.5 \hbar$ and $12.5 \hbar$, which leads to $\mathcal{J}_{1}=14 \hbar^{2} / \mathrm{MeV}$ and $11 \hbar^{2} / \mathrm{MeV}$.

In order to show the effects of pairing correlations on the energy spectra and the wobbling frequency, the results labeled as PRM$^{*}$ are included in Fig. 1. In the calculation, the pairing gap is taken according to the empirical formula $\Delta=12 \mathrm{MeV} / \sqrt{A}$. It is seen that the results without and with paring are very similar in the spin region $I \leqslant 13.5 \hbar$. Hence, the calculated wobbling frequencies at $I \leqslant 12.5 \hbar$ do not change much. With the inclusion of the pairing correlation, the transverse wobbling band ends $2 \hbar$ earlier than the data. 


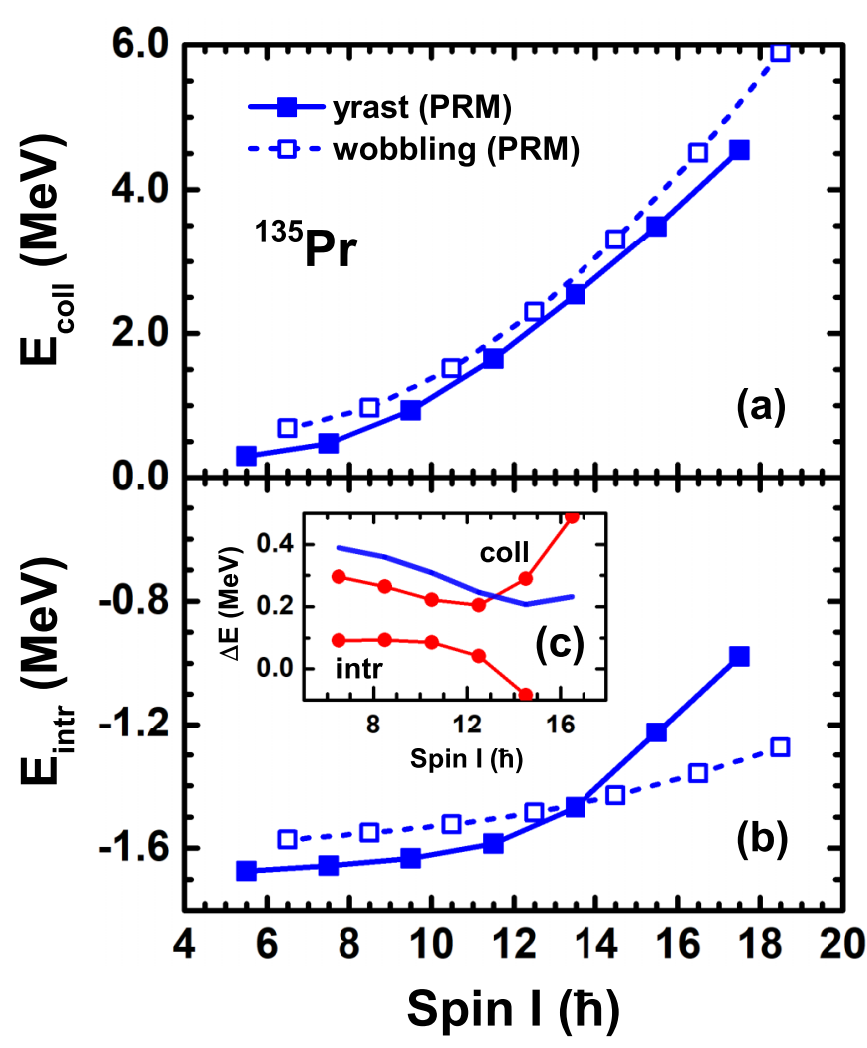

FIG. 2. Energy expectation values of the collective rotor (a) and the valence proton (b) calculated in the PRM for the yrast and wobbling bands in ${ }^{135} \mathrm{Pr}$, together with their differences $\Delta E$ and the full wobbling frequency (c) as functions of the spin $I$.

Therefore, the subsequent discussion is focused on the results without pairing correlations.

In Fig. 2, the separated energy expectation values $E_{\text {coll }}$ and $E_{\text {intr }}$ of the collective rotor Hamiltonian $\hat{H}_{\text {coll }}$ and the intrinsic single-proton Hamiltonian $\hat{H}_{\text {intr }}$ as calculated in the PRM for the yrast and wobbling bands in ${ }^{135} \mathrm{Pr}$ are shown as functions of the spin $I$, together with the differences $\Delta E$ in the two bands and the wobbling frequency.

It is seen that $E_{\text {coll }}$ increases with the spin, and apparently, the yrast band has lower $E_{\text {coll }}$ than the wobbling band. The difference of $E_{\text {coll }}$ in the wobbling and yrast band decreases up to $I=12.5 \hbar$, and then it increases rapidly.

In the region $I \leqslant 11.5 \hbar$, the values of $E_{\text {intr }}$ in the yrast and wobbling bands do not vary much, which implies that the alignment of the proton particle along the $s$ axis remains almost unchanged. This is a specific feature of the wobbling mode in contrast to the signature-partner mode, where the alignment of the single particle varies with the spin $[8,36]$. The values of $E_{\text {intr }}$ in the yrast band are a bit smaller than those in the wobbling band, but their differences stay almost constant. As a consequence, the decrease of the wobbling frequencies originates mainly from the decrease of the $E_{\text {coll }}$ differences.

However, from $I=13.5 \hbar$ upward, $E_{\text {intr }}$ of the yrast band increases rapidly, which is caused by the change of alignment of the proton particle from the $s$ axis towards the $i$ axis, driven by the Coriolis interaction. As revealed by the azimuthal plots (discussed later), this corresponds to a change of the
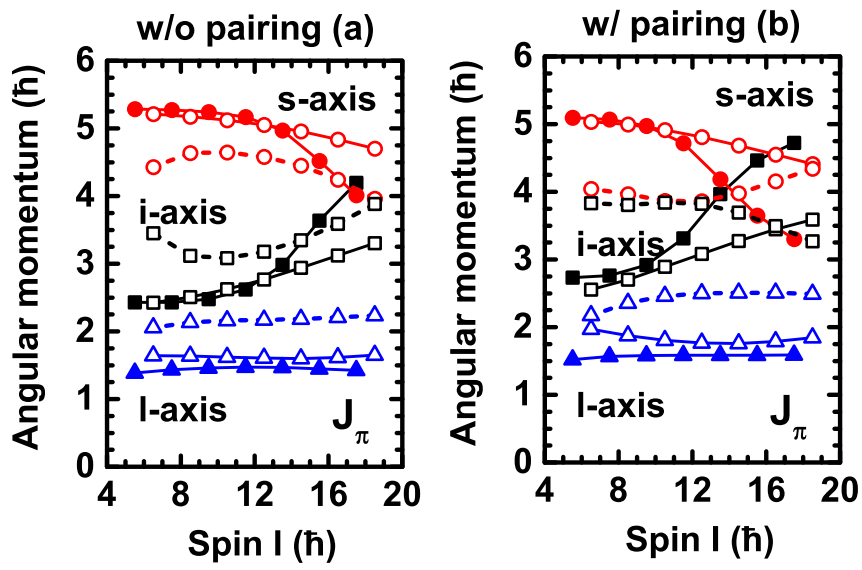

FIG. 3. Root mean square expectation values of the proton angular momentum components along the $s, i$, and $l$ axes as functions of the spin $I$ calculated without (a) and with (b) pairing correlations.

rotational mode from along a principal axis ( $s$ axis) to a planar rotation (with $\boldsymbol{I}$ lying in the si plane). This rearrangement leads to much larger values of $E_{\text {intr }}$ in the yrast band than in the wobbling band, and hence their difference decreases to negative value for $I \geqslant 12.5 \hbar$.

To understand clearly the behaviors of $E_{\mathrm{intr}}$, the root mean square expectation values of the proton particle angular momentum components along the $s, i$, and $l$ axes as functions of spin are shown in Fig. 3(a). It is seen that the proton particle is mainly aligned along the $s$ axis in the region $I \leqslant 13.5 \hbar$, such that $j$ is approximately perpendicular to the $i$ axis. From $I=13.5 \hbar$ upward, the proton particle shows a clear trend to align towards the $i$ axis, corresponding to the increase of $E_{\text {intr }}$.

In addition, the components of $\boldsymbol{j}$ in the signature partner band are shown in Fig. 3(a) by open symbols connected with dashed lines. It can be seen that they are totally different from those of the yrast and wobbling bands. This illustrates the picture that the particle changes its direction of alignment from the yrast band to its signature partner band.

In Fig. 3(b), the analogous results with pairing correlations including are given. It is seen that the alignments of the proton in the yrast and wobbling bands are similar for $I \leqslant 11.5 \hbar$. Due to the pairing correlations, the alignment along the $i$ axis becomes more pronounced and thus the transverse wobbling motion ends earlier. Nevertheless, in both cases, the angular momentum components of proton particle behave very differently in the signature partner band in comparison to yrast and wobbling bands. Therefore, the signature partner band can be distinguished on the basis of these angular momentum components. Here, we should also mention that in the current PRM calculations, the band-head energy of the signature partner band is overestimated by about $900 \mathrm{keV}$ (without pairing correlations) and $500 \mathrm{keV}$ (with pairing correlations) in comparison to the experimental data. This deficiency has also occurred in Refs. [3,15].

\section{B. Azimuthal plot}

The successful reproduction of the energy spectra in the yrast and wobbling bands for ${ }^{135} \mathrm{Pr}$ suggests that the PRM 

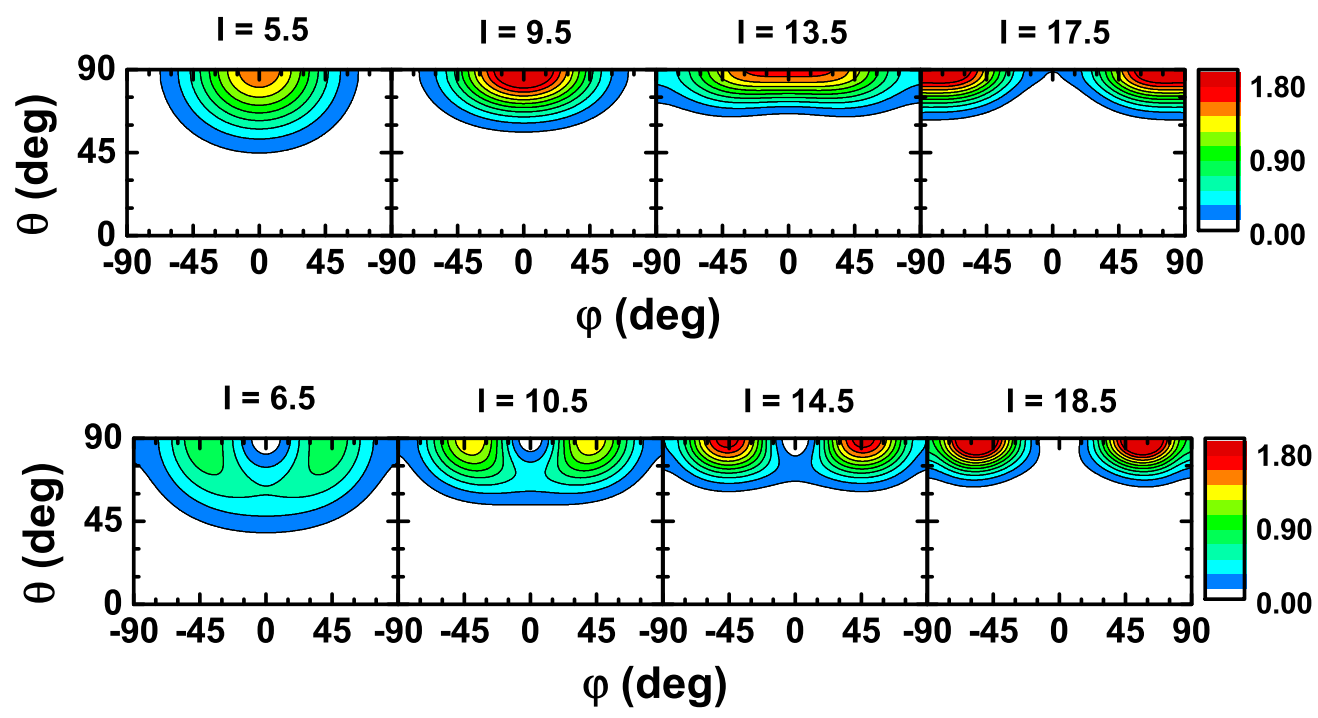

FIG. 4. Azimuthal plots (i.e., distributions of the orientation of the angular momentum) calculated at $I=5.5,9.5,13.5$, and $17.5 \hbar$ for the yrast band and at $I=6.5,10.5,14.5$, and $18.5 \hbar$ for the wobbling band in ${ }^{135} \mathrm{Pr}$.

calculation describes well the wave functions underlying the experimental states. Let us now investigate the angular momentum geometry of the system in detail.

In Fig. 4, the obtained profiles $\mathcal{P}(\theta, \varphi)$ for the orientation of the angular momenta $\boldsymbol{I}$ in the $\theta \varphi$ plane are shown at spin $I=5.5,9.5,13.5$, and $17.5 \hbar$ for the yrast band, and at $I=$ $6.5,10.5,14.5$, and $18.5 \hbar$ for the wobbling band in ${ }^{135} \mathrm{Pr}$. We remind the reader that $\theta$ is the angle between the $I$ and the $l$ axis, and $\varphi$ is the angle between the projection of $\boldsymbol{I}$ onto the si plane and the $s$ axis.

One observes that the maximum of $\mathcal{P}(\theta, \varphi)$ is always located at $\theta=90^{\circ}$. This is because the $l$ axis carries the smallest MoI, and in order to lower the energy the angular momentum prefers to lie in the si plane. Note that due to the $D_{2}$ symmetry, $\mathcal{P}(\theta, \varphi)$ is an even function of $\varphi$. For the states in the yrast band, the $\varphi$ coordinates of the maxima gradually deviate from zero with increasing spin. As a result, the number of maxima changes from one to two. This implies that the rotational mode in the yrast band changes from a principal axis rotation at the low spins $(I=5.5$ and $9.5 \hbar)$ to a planar rotation at high spins $(I=13.5 \hbar)$. By examining the profiles $\mathcal{P}(\theta, \varphi)$ for all yrast states, we find that $I=13.5 \hbar$ is the critical spin at which the rotational mode changes (with $\varphi \simeq \pm 5^{\circ}$ at the maxima). At $I=17.5 \hbar$, the $\varphi$ coordinates of the maxima of $\mathcal{P}(\theta, \varphi)$ approaches $\pm 90^{\circ}$. In this case, the rotational mode changes from a planar rotation back to a principal axis rotation about the $i$ axis. These features are similar to the behavior of the minima of the total Routhian surface as a function of the rotational frequency, calculated by TAC in the Refs. [6,37]. Both PRM and TAC present the same physics picture: a principal axis rotation about the $s$ axis at low spins, a transition to planar rotation at intermediate spins, and a return to principal axis rotation about the $i$ axis at high spins.

In the lower part of Fig. 4, the distributions $\mathcal{P}(\theta, \varphi)$ exhibit a different behavior in the wobbling band. With one-phonon excitation (wobbling motion), the profiles $\mathcal{P}(\theta, \varphi)$ have two maxima for all spins. At low spins $(I \leqslant 12.5 \hbar)$, the excitation is transverse wobbling about the $s$ axis. This is reflected by the larger $\varphi$ values of the maxima of $\mathcal{P}(\theta, \varphi)$ in wobbling states (with spin $I$ ) compared to those of the corresponding yrast states (with spin $I-1$ ). Note that for the zero-phonon states (with $I \leqslant 11.5 \hbar$ ) the underlying wave functions are symmetric and peaked at $\varphi=0^{\circ}$ ( $s$ axis), whereas for onephonon states $(I=6.5 \hbar, 8.5 \hbar$, etc.) they are antisymmetric and have a node at $\varphi=0^{\circ}$. At high spins $(I \geqslant 17.5 \hbar)$, the excitation from the yrast band into the wobbling band is longitudinal wobbling about the $i$ axis. This is in accordance with the fact that the $\varphi$ coordinate of the maxima of $\mathcal{P}(\theta, \varphi)$ in the wobbling states (with spin $I$ ) are smaller than those in the yrast states (with spin $I-1$ ). Moreover, the zero-phonon state $(I=17.5 \hbar)$ is peaked at $\varphi= \pm 90^{\circ}$ ( $i$ axis), while the one-phonon state ( $I=18.5 \hbar$ ) has a node there. These features are similar to the properties obtained with wave functions calculated from a collective Hamiltonian in Refs. [6,37].

Therefore, we have confirmed that with the increasing spin, the wobbling mode varies from the transverse at low spins to longitudinal at high spins, which is consistent with the evolution of the wobbling frequency in Fig. 1. In fact, this variation is mainly driven by the collective rotor (cf. Fig. 2).

\section{C. $R$ plots}

According to the above analysis, the collective rotor plays an essential role in the wobbling motion. Therefore, we investigate in the following the probability distribution of the rotor angular momentum ( $R$ plots) as well as the its projections onto each principal axis ( $K_{R}$ plots).

In Fig. 5, the probability distributions $P_{R}$ of the rotor angular momentum ( $R$ plots) calculated by Eq. (22) are displayed for the yrast and wobbling bands in ${ }^{135} \mathrm{Pr}$. For a given spin $I$, the integer $R$ takes values from $|I-j|$ to $I+j$, excluding $R=1$. It is found that for all $I$ the probability $P_{R}$ almost 

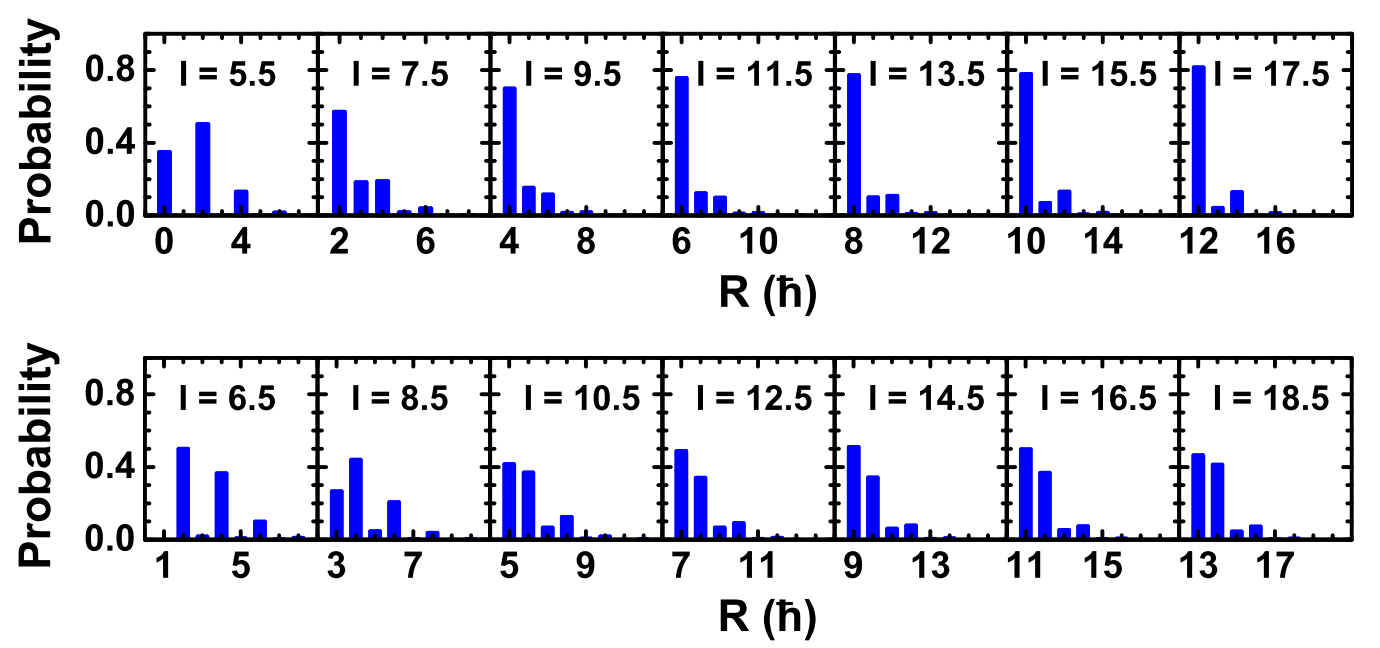

FIG. 5. The probability distributions for the angular momentum of rotor ( $R$ plots) for the yrast and wobbling bands in ${ }^{135} \operatorname{Pr}$.

vanishes for large $R$. Therefore, the $R$ plots are restricted in Fig. 5 to small $R$.

For the yrast band, $P_{R}$ has a pronounced peak at $R_{\min }=$ $|I-j|$, except for $I=5.5 \hbar$ (the band head), where the maximal weight occurs at $R=|I-j|+2=2 \hbar$. For the wobbling band, $P_{R}$ has two peaks of similar height, which are located at $R=|I-j|$ and $|I-j|+1$. An exception is again the band head $I=6.5 \hbar$, where the peaks lie at $R=|I-j|+1$ and $|I-j|+3$. The $R$ plots indicate that $R$ is an asymptotic good quantum number in the yrast band $(I \geqslant 7.5 \hbar)$, but not in the wobbling band. This is different to the wobbling motion of a pure triaxial rotor, where $R$ is a good quantum in all bands $[2,38]$. However, it should be noted that the admixture of the states with $R=|I-j|$ and $R=\mid I-$ $j \mid+1$ in the wobbling band is important as it provides the possibility for the (quantum mechanical) wobbling transition. This admixture causes that the average value of $R$ in the wobbling band $R_{\text {wobb }}(I)$ at spin $I$ is larger than $|I-j|$ and leads to $R_{\text {wobb }}(I)-R_{\text {yrast }}(I-1)>1 \hbar$, so that the rotor in the wobbling band with spin $I$ has to wobble to increase its spin by only $1 \hbar$ with respect to the yrast band (with spin $I-1$ ).

\section{D. $K_{R}$ plots}

In the following the probability distributions for the projections $\left(K_{R}=R_{l}, R_{s}\right.$, and $R_{i}$ ) of the rotor angular momentum onto the $l, s$, and $i$ axes ( $K_{R}$ plots) will be investigated. For the triaxiality parameter $\gamma=-26^{\circ}$, the $l$ axis is the designated quantization axis. The distributions with respect to the $s$ and $i$ axes are obtained by taking $\gamma=146^{\circ}$ and $266^{\circ}$, respectively. These $\gamma$ values correspond to the equivalent sectors such that the nuclear shape remains the same, but only the principal axes are interchanged [20].

In Fig. 6, the probability distributions for the projection of the rotor angular momentum onto the $l$ axis $P_{R_{l}}$ as calculated in the PRM, are shown for the yrast and wobbling bands in ${ }^{135} \mathrm{Pr}$. For both the yrast and wobbling bands, $P_{R_{l}}$ has two peaks at $R_{l}=0$ and $2 \hbar$, indicating that the rotor angular momentum has only very small components along the $l$ axis, to which a very small $\mathrm{MoI}$ is associated. This is consistent with the azimuthal plots shown in Fig. 4. At the same time, the distributions of $P_{R_{l}}$ for the yrast and the wobbling bands do not change much as the spin $I$ increases, indicating that the
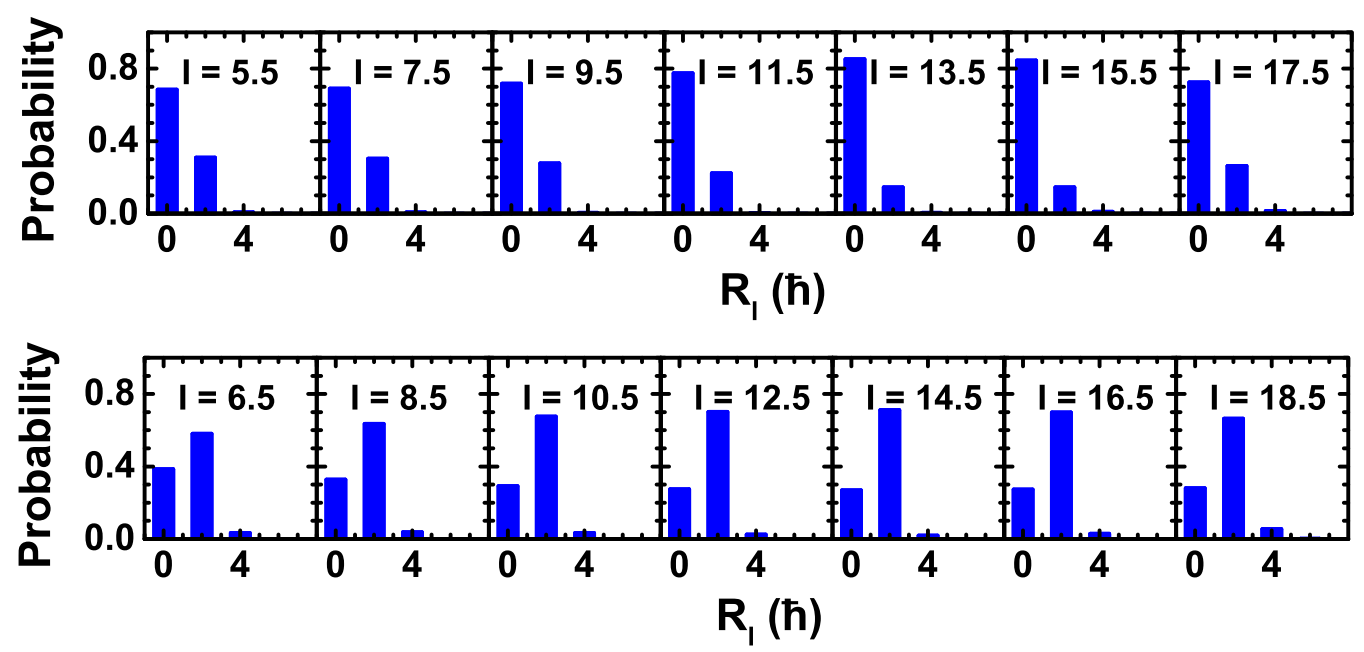

FIG. 6. Probability distributions for the projection of the rotor angular momentum onto the $l$ axis for the yrast and wobbling bands in ${ }^{135} \mathrm{Pr}$. 

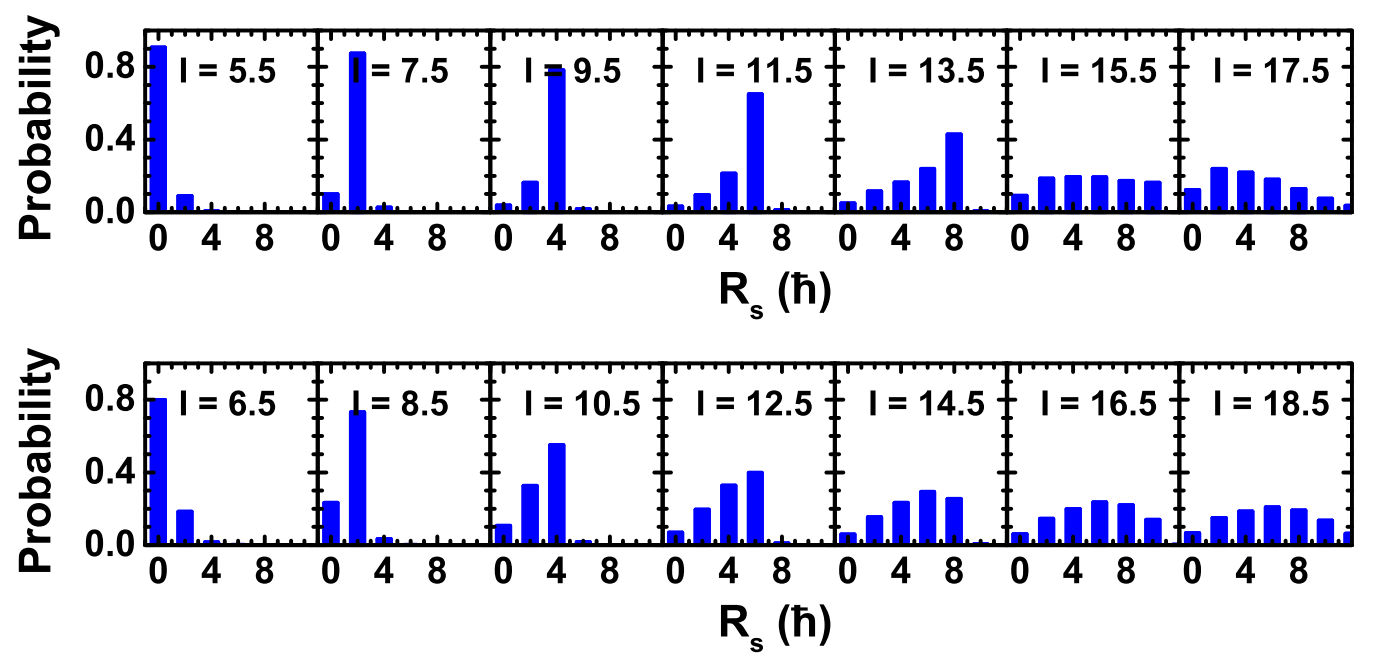

FIG. 7. Same as Fig. 6, but for the projection onto the $s$ axis.

rotor angular momentum component along the $l$ axis remains almost constant. For the yrast band, $P_{R_{l}}$ at $R_{l}=0 \hbar$ is much larger than $P_{R_{l}}$ at $R_{l}=2 \hbar$, while for the wobbling band, the situation is opposite. There, $P_{R_{l}}$ at $R_{l}=2 \hbar$ is larger than $P_{R_{l}}$ at $R_{l}=0 \hbar$.

The probability distributions $P_{R_{s}}$ of the component $R_{s}$ are displayed in Fig. 7 for the yrast and wobbling bands in ${ }^{135} \mathrm{Pr}$. In the region $I \leqslant 13.5 \hbar$, the distributions $P_{R_{s}}$ for states in the yrast band (with $I-1$ ) and the wobbling band (with $I$ ) show a similar behavior. This indicates that the rotor angular momenta of states in the yrast (with $I-1$ ) and wobbling (with $I$ ) bands have similar components along the $s$ axis due to the transverse wobbling motion. For neighboring states with $I-2$ and $I$, the distance between the peaks of $P_{R_{s}}$ is $2 \hbar$. In the region $I \geqslant 14.5 \hbar$, where the transverse wobbling motion disappears, the distributions $P_{R_{S}}$ are spread over many $R_{S}$ values. The average value of $R_{s}$ is about $4 \hbar$ for the yrast band and about $6 \hbar$ for the wobbling band.

In Fig. 8, the probability distributions $P_{R_{i}}$ of the component $R_{i}$ are shown for the yrast and wobbling bands in ${ }^{135} \mathrm{Pr}$.
In comparison to $P_{R_{l}}$ and $P_{R_{s}}$, the distributions $P_{R_{i}}$ reveal stronger admixtures of the various values of $R_{i}$, which originates from the wobbling motion of the rotor towards the $i$ axis. One also observes that $P_{R_{i}}$ of the yrast and wobbling bands behavior differently. In the region $I \leqslant 13.5 \hbar$, the probability $P_{R_{i}}$ at $R_{i}=0 \hbar$ has a finite value in the yrast band, while it vanishes for the wobbling band. This is a characteristic of the one-phonon excitation of the wobbling motion. Namely, the underlying wave function for a zero-phonon state (yrast band) is even under $R_{i} \rightarrow-R_{i}$, whereas for a one-phonon state (wobbling band) it is odd. This picture is also consistent with the features displayed in the azimuthal plots (cf. Fig. 4). The peak position of the distribution $P_{R_{i}}$ increases by about $2 \hbar$ from a state in the yrast band (with $I-1$ ) to a state in the wobbling band (with $I$ ). This increment is caused by the wobbling motion from the $s$ axis towards the $i$ axis. For neighboring states with $I-2$ and $I$, the average value of $R_{i}$ differs by about $1 \hbar$. This means that $R_{i}$ for the state $I$ in the yrast band is about $1 \hbar$ smaller than for the state $I-1$ in the wobbling band.
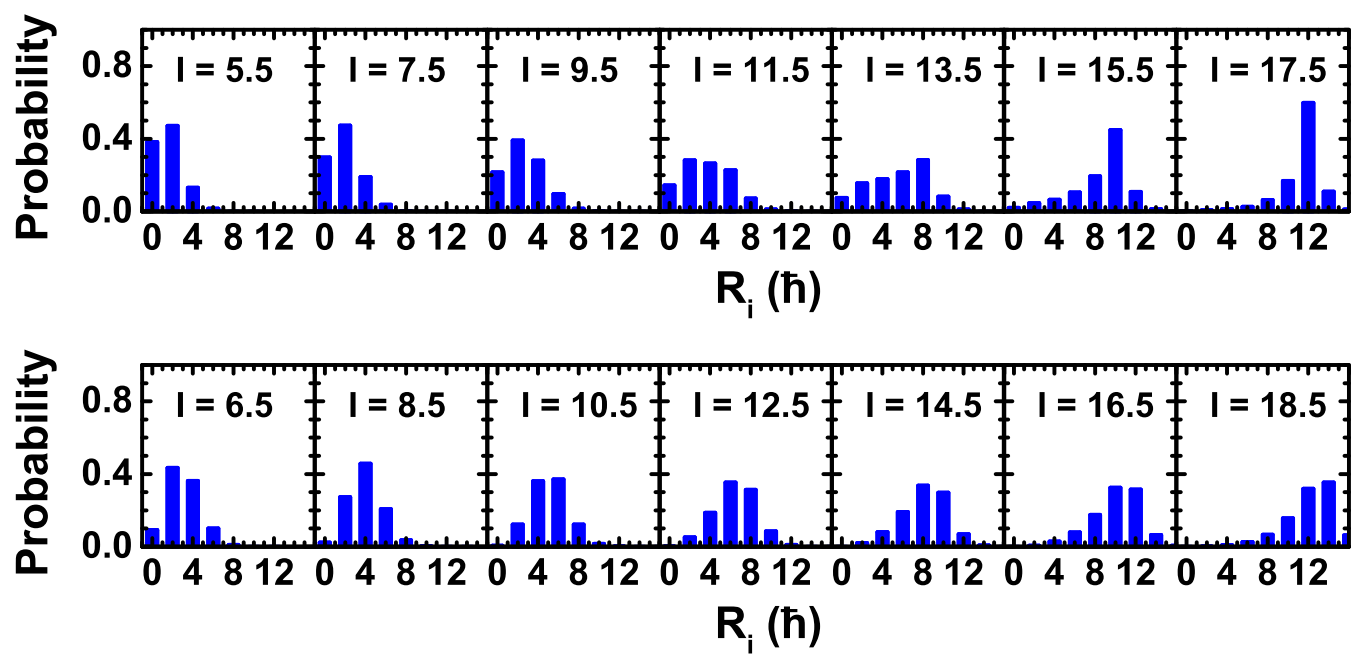

FIG. 8. Same as Fig. 6, but for the projection onto the $i$ axis. 
In the region $I \geqslant 14.5 \hbar$, the distributions $P_{R_{i}}$ for the yrast band show a clear peak at $R_{i}=|I-j|=R$ (cf. Figs. 5 and 8 ), indicating that the rotor has aligned with the $i$ axis. For the wobbling band one observes two peaks of similar height at $R_{i}=|I-j|-1$ and $|I-j|+1$, which gives an increment of $R_{i}$ by about $1 \hbar$ from the yrast state (with $I-1$ ) to the wobbling state (with $I$ ). This behavior is different from the transverse wobbling region, where the increment is about $2 \hbar$.

\section{E. Angular momentum coupling schemes}

From the above analysis of energy expectation values of the intrinsic Hamiltonian $\hat{H}_{\text {intr }}$, azimuthal plots $\mathcal{P}(\theta, \varphi)$ of the total angular momentum, and the $R$ plots and three $K_{R}$ plots for the rotor angular momentum, one can deduce the following features in the transverse wobbling region:

(i) the single-particle (angular momentum) is aligned with the $s$ axis;

(ii) the average rotor angular momentum is more than $1 \hbar$ (and less than $2 \hbar$ ) longer in the wobbling band with spin $I+1$ than in the yrast band with spin $I$;

(iii) the projection of the rotor angular momentum onto the $l$ axis is very small;

(iv) the rotor angular momenta in yrast states (with $I$ ) and wobbling states (with $I+1$ ) have similar components along the $s$ axis. For neighboring states with $I-2$ and $I$, the component $R_{s}$ differs by about $2 \hbar$;

(v) the component $R_{i}$ increases by about $2 \hbar$ from an yrast state $I$ to a wobbling state $I+1$. In addition, $R_{i}$ in the yrast state $I$ is about $1 \hbar$ smaller than its value in the wobbling state $I-1$.

Combining these features, a schematic illustration of the coupling scheme of the angular momenta $\boldsymbol{j}$ and $\boldsymbol{R}$, of the high- $j$ particle and the rotor, for transverse wobbling in an yrast state $I$ and two wobbling states $I \pm 1$ is shown in Fig. 9.

On the other hand, for longitudinal wobbling one finds the following features:

(i) the proton particle (angular momentum) is aligned with the $i$ axis;

(ii) the average value of $R_{s}$ is about $4 \hbar$ in the yrast band and about $6 \hbar$ in the wobbling band.

(iii) the increment of $R_{i}$ from an yrast state with $I-1$ to a wobbling state with $I$ is about $1 \hbar$.

Again combining these features, a schematic illustration of the coupling scheme of $\boldsymbol{j}$ and $\boldsymbol{R}$ for the longitudinal wobbling motion in an yrast state with $I$ and two wobbling states with $I \pm 1$ is shown in Fig. 10. This coupling scheme differs from that for transverse wobbling, shown in Fig. 9. One can clearly see that the rotor angular momentum is much longer than the single particle angular momentum. It should be noted that a schematic illustration of the longitudinal wobbling motion has also been given in Refs. [8,36], but there the MoI belonging to $s$ axis was assumed to be the largest. In that case, the angular momenta of the rotor and the particle both align with the $s$ axis in the yrast band.

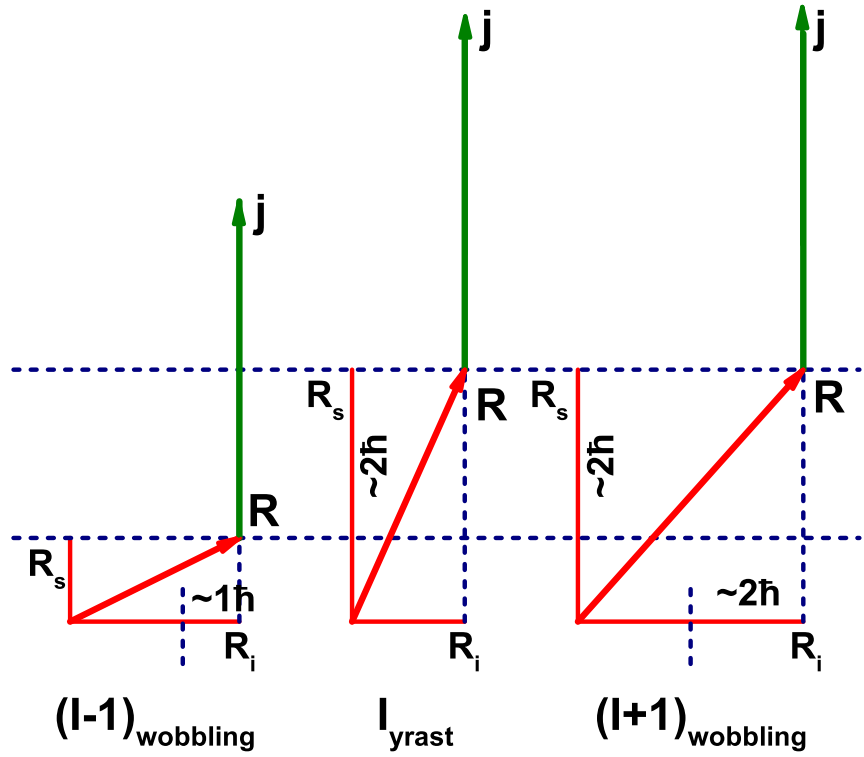

FIG. 9. Schematic illustration of the coupling scheme of the angular momenta $\boldsymbol{j}$ and $\boldsymbol{R}$ of the high- $j$ particle and the rotor for the transverse wobbling in an yrast state with $I$ and two wobbling states with $I \pm 1$. The total angular momentum is $\boldsymbol{I}=\boldsymbol{R}+\boldsymbol{j}$.

\section{SUMMARY}

In summary, the behavior of the collective rotor for the wobbling motion of ${ }^{135} \mathrm{Pr}$ has been investigated in the PRM. After successful reproduction of the experimental energy spectra and the wobbling frequencies, the separate contributions from the rotor and the single-particle Hamiltonian to the wobbling frequencies have been analyzed. It is confirmed that the collective rotor motion is responsible for the decrease of the wobbling frequency in transverse wobbling, and its increase in longitudinal wobbling found in Refs. [3,5,6].

The evolution of the wobbling mode in ${ }^{135} \mathrm{Pr}$ from transverse at low spins to longitudinal at high spins has been illustrated by the distributions $\mathcal{P}(\theta, \varphi)$ of the total angular momentum in the intrinsic frame (azimuthal plots). According to the analysis of the probability distributions of the rotor angular momentum ( $R$ plots) and their projections onto the three principal axes ( $K_{R}$ plots), different schematic coupling schemes of the angular momenta $\boldsymbol{j}$ and $\boldsymbol{R}$ of the rotor and the high- $j$ particle in the transverse and longitudinal wobbling have been obtained.

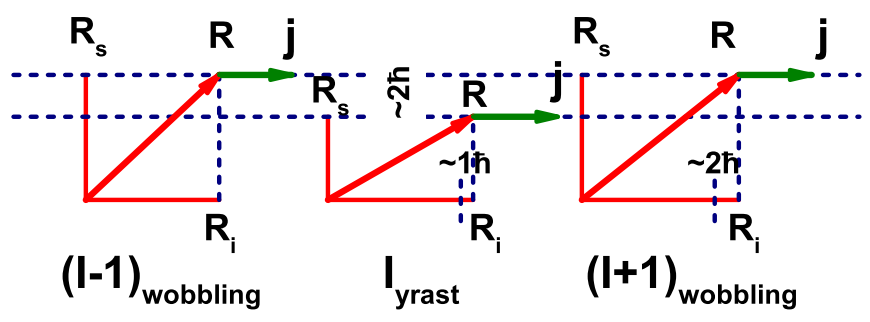

FIG. 10. Similar as Fig. 9, but for the longitudinal wobbling motion. 
In perspective, the $R$ plots and $K_{R}$ plots presented in this work can be used to examine the fingerprints of electromagnetic transitions ( $E 2$ or $M 1)$ between wobbling bands, or can be extended to investigate, e.g., the behavior of the collective rotor for chiral rotation [1].

Finally, we would like to discuss the $B(M 1)$ values in the PRM. With inclusion of pairing correlations, the $B(M 1)$ values can be reduced by a factor of 2-3 compared to those without pairing correlations. However, this $B(M 1)$ values still overestimate the experimental ones by a factor of 3-10. This problem has been interpreted in Ref. [39] as follows: the wobbling motion is not a pure orientation vibration of the rigid quadrupole mass tensor with respect to the angular momentum vector, but includes also a coupling to vibrations of the proton and neutron currents against each other. However, this scissor mode is not taken into account in the PRM and hence leads an overestimation of $B(M 1)$. The scissor mode does not have any obvious influence on the energy spectra and interband $B(E 2)$ values. In order to cure this problem, a quenched $g$ factor was used in Ref. [18] to reproduce the data in the wobbling bands of ${ }^{135} \mathrm{Pr}$. A satisfactory solution of this problem in the PRM with separated proton and neutron rotors requires more efforts.

\section{ACKNOWLEDGMENTS}

One of the authors (Q.B.C.) thanks S. Frauendorf for helpful discussions. Financial support for this work was provided by Deutsche Forschungsgemeinschaft (DFG) and National Natural Science Foundation of China (NSFC) through funds provided to the Sino-German CRC 110 "Symmetries and the Emergence of Structure in QCD" (DFG Grant No. TRR110 and NSFC Grant No. 11621131001). The work of U.G.M. was also supported by the Chinese Academy of Sciences (CAS) President's International Fellowship Initiative (PIFI) (Grant No. 2018DM0034) and by VolkswagenStiftung (Grant No. 93562).
[1] S. Frauendorf and J. Meng, Nucl. Phys. A 617, 131 (1997).

[2] A. Bohr and B. R. Mottelson, Nuclear Structure (Benjamin, New York, 1975), Vol. II.

[3] S. Frauendorf and F. Dönau, Phys. Rev. C 89, 014322 (2014).

[4] M. Matsuzaki, Y. R. Shimizu, and K. Matsuyanagi, Phys. Rev. C 65, 041303(R) (2002).

[5] Y. R. Shimizu, M. M. Matsuzaki, and K. Matsuyanagi, arXiv:0404063v1[nucl-th].

[6] Q. B. Chen, S. Q. Zhang, and J. Meng, Phys. Rev. C 94, 054308 (2016).

[7] P. Bringel, G. B. Hagemann, H. Hübel, A. Al-khatib, P. Bednarczyk, A. Bürger, D. Curien, G. Gangopadhyay, B. Herskind, D. R. Jensen et al., Eur. Phys. J. A 24, 167 (2005).

[8] S. W. Ødegård, G. B. Hagemann, D. R. Jensen, M. Bergström, B. Herskind, G. Sletten, S. Törmänen, J. N. Wilson, P. O. Tjøm, I. Hamamoto et al., Phys. Rev. Lett. 86, 5866 (2001).

[9] D. R. Jensen, G. B. Hagemann, I. Hamamoto, S. W. Ødegård, B. Herskind, G. Sletten, J. N. Wilson, K. Spohr, H. Hübel, P. Bringel et al., Phys. Rev. Lett. 89, 142503 (2002).

[10] G. Schönwaßer, H. Hübel, G. B. Hagemann, P. Bednarczyk, G. Benzoni, A. Bracco, P. Bringel, R. Chapman, D. Curien, J. Domscheit et al., Phys. Lett. B 552, 9 (2003).

[11] H. Amro, W. C. Ma, G. B. Hagemann, R. M. Diamond, J. Domscheit, P. Fallon, A. Gorgen, B. Herskind, H. Hübel, D. R. Jensen et al., Phys. Lett. B 553, 197 (2003).

[12] D. J. Hartley, R. V. F. Janssens, L. L. Riedinger, M. A. Riley, A. Aguilar, M. P. Carpenter, C. J. Chiara, P. Chowdhury, I. G. Darby, U. Garg et al., Phys. Rev. C 80, 041304(R) (2009).

[13] S. J. Zhu, Y. X. Luo, J. H. Hamilton, A. V. Ramayya, X. L. Che, Z. Jiang, J. K. Hwang, J. L. Wood, M. A. Stoyer, R. Donangelo et al., Int. J. Mod. Phys. E 18, 1717 (2009).

[14] Y. X. Luo, J. H. Hamilton, A. V. Ramayya, J. K. Hwang, S. H. Liu, J. O. Rasmussen, S. Frauendorf, G. M. Ter-Akopian, A. V. Daniel, and Y. T. Oganessian, Exotic nuclei: Exon-2012: Proceedings of the International Symposium, 215 (2013).

[15] J. T. Matta, U. Garg, W. Li, S. Frauendorf, A. D. Ayangeakaa, D. Patel, K. W. Schlax, R. Palit, S. Saha, J. Sethi et al., Phys. Rev. Lett. 114, 082501 (2015).
[16] S. Biswas et al., arXiv:1608.07840[nucl-ex].

[17] J. A. Sheikh, G. H. Bhat, W. A. Dar, S. Jehangir, and P. A. Ganai, Phys. Scr. 91, 063015 (2016).

[18] K. Tanabe and K. Sugawara-Tanabe, Phys. Rev. C 95, 064315 (2017).

[19] R. Budaca, Phys. Rev. C 97, 024302 (2018).

[20] P. Ring and P. Schuck, The Nuclear Many Body Problem (Springer Verlag, Berlin, 1980).

[21] A. Faessler and H. Toki, Phys. Lett. B 59, 211 (1975).

[22] H. Toki and A. Faessler, Phys. Lett. B 63, 121 (1976).

[23] H. A. Smith and F. A. Rickey, Phys. Rev. C 14, 1946 (1976).

[24] I. Ragnarsson and P. B. Semmes, Hyperfine Interact. 43, 425 (1988).

[25] A. Mukherjee, U. Datta Pramanik, M. S. Sarkar, and S. Sen, Phys. Rev. C 50, 1868 (1994).

[26] S. Modi, M. Patial, P. Arumugam, E. Maglione, and L. S. Ferreira, Phys. Rev. C 95, 024326 (2017).

[27] F. Dönau and S. Frauendorf, Phys. Lett. B 71, 263 (1977).

[28] S. Quan, W. P. Liu, Z. P. Li, and M. S. Smith, Phys. Rev. C 96, 054309 (2017).

[29] H. Esbensen and C. N. Davids, Phys. Rev. C 63, 014315 (2000).

[30] C. N. Davids and H. Esbensen, Phys. Rev. C 69, 034314 (2004).

[31] S. Modi, M. Patial, P. Arumugam, E. Maglione, and L. S. Ferreira, Phys. Rev. C 95, 054323 (2017).

[32] S. Modi, M. Patial, P. Arumugam, L. S. Ferreira, and E. Maglione, Phys. Rev. C 96, 064308 (2017).

[33] S. Y. Wang, B. Qi, and S. Q. Zhang, Chin. Phys. Lett. 26, 052102 (2009).

[34] F. Q. Chen, Q. B. Chen, Y. A. Luo, J. Meng, and S. Q. Zhang, Phys. Rev. C 96, 051303(R) (2017).

[35] Q. B. Chen and J. Meng, Phys. Rev. C 98, 031303(R) (2018).

[36] I. Hamamoto, Phys. Rev. C 65, 044305 (2002).

[37] Q. B. Chen, S. Q. Zhang, P. W. Zhao, and J. Meng, Phys. Rev. C 90, 044306 (2014).

[38] W. X. Shi and Q. B. Chen, Chin. Phys. C 39, 054105 (2015).

[39] S. Frauendorf and F. Dönau, Phys. Rev. C 92, 064306 (2015). 